\title{
Globe
}

Revue internationale d'études québécoises

Paul Beaucage, Gilles Groulx, le cinéaste résistant, Lux Éditeur, Montréal, 2009

\section{Germain Lacasse}

Volume 13, numéro 2, 2010

URI : https://id.erudit.org/iderudit/1001140ar

DOI : https://doi.org/10.7202/1001140ar

Aller au sommaire du numéro

Éditeur(s)

Globe, Revue internationale d'études québécoises

ISSN

1481-5869 (imprimé)

1923-8231 (numérique)

Découvrir la revue

Citer ce compte rendu

Lacasse, G. (2010). Compte rendu de [Paul Beaucage, Gilles Groulx, le cinéaste résistant, Lux Éditeur, Montréal, 2009]. Globe, 13(2), 204-209.

https://doi.org/10.7202/1001140ar d'utilisation que vous pouvez consulter en ligne.

https://apropos.erudit.org/fr/usagers/politique-dutilisation/ 
et de la place qui lui est conférée dans le système scolaire depuis les trente dernières années.

L'ouvrage se clôt par une cinquième partie, plus prospective, constituée d'une série d'articles qui ouvrent la réflexion et projettent le débat dans l'avenir, cela à partir de réflexions pourtant guidées par la lecture d'auteurs que l'on pourrait déjà qualifier de "classiques» en sciences sociales.

Au final, on pourra dire que ce volume n'est pas une simple contribution de plus sur le phénomène religieux en contexte québécois. A l'inverse, si la perspective pluridisciplinaire de l'ouvrage ouvre l'horizon du lecteur, on soulignera également l'intérêt de réunir dans un même volume les contributions d'auteurs - jeunes chercheurs ou auteurs dont l'expertise est déjà plus reconnue - défendant des positions parfois antagonistes dans le débat social. Il ne s'agit donc pas d'un livre qui clôt la réflexion, mais bel et bien d'un livre qui élargit les perspectives en proposant des outils divers et variés au lecteur soucieux de comprendre les transformations de la société québécoise sans en omettre l'un des principaux facteurs: le rapport des Québécois au religieux.

David Koussens

European University Institute (Italie)

\section{Paul Beaucage \\ Gilles Groulx, le cinéaste résistant, Lux Éditeur, Montréal, 2009.}

Un livre ordinaire sur un cinéaste exceptionnel. Gilles Groulx mérite certainement mieux. On lui avait déjà consacré des études, que l'auteur ne discute ni ne cite, pas plus qu'il ne cite les importants ouvrages sur le cinéma québécois qui traitent de Groulx et son œuvre. Au final il reste un livre un peu bancal; invitation à faire mieux, ce ne sera pas difficile.

L'ouvrage de Beaucage est divisé en quinze chapitres, suivant les quinze films réalisés par Groulx. Ces quinze films sont répartis en trois parties: les années d'apprentissage, l'émergence d'un grand cinéaste, liberté et restriction de l'engagement politique. Cette périodisation est discutable, mais passons pour l'instant. Le tout est amorcé par une trop brève introduc- 
tion qui rappelle l'origine et les traits du protagoniste. Né dans un milieu ouvrier modeste, Groulx avait un grand talent de dessinateur qui le guide vers des études à l'école des Beaux Arts, à l'époque du Refus Global qui l'influence profondément. Ami du poète Claude Gauvreau et passionné comme lui de littérature et de cinéma, il publie un recueil de poèmes et tourne un film amateur. Le premier chapitre rappelle avec intérêt ce film que Groulx tourne pendant ses études, avec une caméra achetée par son frère: Les héritiers. Beaucage en parle comme d'un film sur les inégalités sociales à Montréal, une «mosaïque visuelle sur les déshérités de la métropole». (p. 22) Il explique que le film souffrait de l'inexpérience et du manque de moyens de l'auteur, mais affichait déjà un sens du rythme et du mouvement. Après ses études Groulx devient monteur de films à Radio-Canada, et c'est en tant que monteur qu'il fera ensuite ses premières armes à l'ONF.

Le deuxième chapitre décrit le premier film réalisé en 1958 à l'ONF avec Michel Brault, œuvre dont Groulx fit le montage à l'insu de ses patrons. Les raquetteurs, devenu le film phare et fondateur du cinéma direct et de l'équipe française de l'Office, décrit de façon sarcastique un rassemblement sportif qui exprimait la décrépitude du Québec colonial et duplessiste. Beaucage dit de ce film qu'il est la vision de Brault et le rythme de Groulx, et l'associe au "réalisme critique» (p. 31) qui marquera l'œuvre de ce dernier. Le troisième chapitre commente Normétal (1959) et les premiers démêlés de Groulx avec la censure interne de l'Office. Le réalisateur voulait montrer la dure réalité de la vie de mineur, mais ses patrons exigeaient des coupures qui firent du film une description candide d'une petite ville minière. Ici le point de vue du biographe est ambigu : tandis que Groulx a renié ce film, il lui reproche d'avoir réalisé une œuvre naïve. Il répétera ces reproches injustifiés, et parsème son texte de citations et de comparaisons qui semblent souvent impertinentes et agacent tout au long de la lecture: Normétal est comparé à Lumière d'été de Jean Grémillon, Où êtes vous donc? à L'homme d'Aran de Flaherty, Première question sur le bonheur à Voici le temps des assassins de Duvivier, etc. Ces comparaisons semblent surtout décoratives, comme les nombreuses citations qui n'apportent rien à l'argumentation ni à l'approche théorique, comme quand il écrit que Groulx refuse «de se complaire dans un réalisme de surface semblable à celui de William Bouguereau, le peintre pompier» (p. 89). C'est comme dire qu'Elvis Presley voulait se démarquer de Claude Debussy!

Le quatrième chapitre commente le film La France sur un caillou, coréalisé avec Claude Fournier en 1960 et consacré aux Îles Saint-Pierre-etMiquelon, seuls territoires français d'Amérique du Nord. Beaucage dit que 
ce film conventionnel et naïf est surtout celui de Claude Fournier, mais il en tient Groulx comme autant responsable (p. 43). Le cinquième chapitre traite de Golden Gloves (1961) ; l'auteur inclut ce film novateur et remarquable dans la partie "Années d'apprentissage» alors que l'œuvre exprime une réelle maîtrise. Le film décrit les rêves et la réalité de boxeurs amateurs noirs et canadiens-français qui rêvent de sortir du rang en remportant un important tournoi. Beaucage écrit que Groulx veut «démystifier un univers trop souvent idéalisé par les masses» (p. 52). Il néglige un peu les aspects sociaux du sujet, mais il décrit bien l'esthétique rapide et alerte du film où une caméra mobile, une musique syncopée et un montage très rythmé font de cette œuvre une danse filmée autant qu'une réflexion sur la lutte des classes et des minorités subalternes.

Le sixième chapitre étudie Voir Miami (1963) dans lequel Groulx essaie de comprendre l'attrait que cette ville exerce dans l'imaginaire québécois. Beaucage apprécie les qualités esthétiques du film, mais le ton est ici un peu condescendant: comme dans le cas de Normétal, l'auteur rappelle que le film a été censuré par l'Office mais reproche à Groulx de n'avoir pas su contourner cette censure: "Évidemment, Groulx n'a pas bénéficié de la liberté esthétique voulue pour mettre en scène son ouvre. Pourtant, on aurait souhaité qu'il se montre plus astucieux afin de traiter, de façon directe ou indirecte, d'une thématique universelle» (p. 63). Le chapitre sept clôt la section "Années d'apprentissage" par un commentaire sur Un jeu si simple (1964) même s'il s'agit d'un autre film important et que l'auteur y souligne la maîtrise atteinte par Groulx dans le discours autant que dans l'esthétique. Comme dans Golden Gloves, Groulx y déconstruit de façon systématique les différents aspects du sport national canadien, s'intéressant aux spectateurs autant qu'au jeu et cherchant à en exposer les fonctions cathartiques ou identitaires. Beaucage associe le film aux réflexions de Bertolt Brecht; chacun sait que Groulx s'y référait, mais l'auteur du livre passe rapidement même s'il y revient souvent. C'est le principal défaut de ce livre, qui remplace l'explication théorique par les litanies de citations incongrues.

Le chapitre huit introduit la section "L'émergence d'un grand cinéaste" par une étude assez fouillée du Chat dans le sac (1964). Premier film de fiction de Groulx et son œuvre la plus connue, il raconte la relation hésitante et tronquée entre une juive anglophone et un jeune "Canadien français " qui se cherche. Le style est proche de la Nouvelle Vague: noir et blanc, caméra mobile, acteurs non professionnels, adresse à la caméra. Le ton est particulièrement pertinent, interrogation sur le devenir d'un personnage qui pose les questions que se posent alors les intellectuels de sa génération en 
pleine "Révolution tranquille». Mais l'analyse de Beaucage est souvent malhabile, dérapant encore et encore vers des commentaires plutôt décalés. Le chapitre neuf passe rapidement sur un film de commande fait par Groulx et Gérald Godin pour l'Office du film du Québec en 1967: Québec... ? qui vantait l'évolution récente et le potentiel du Québec moderne.

Le chapitre dix aborde Où êtes vous donc? (1968) film insolite et provocateur sur la révolte de jeunes Québécois qui tentent d'inventer entre eux de nouvelles relations mais se séparent faute de trouver des valeurs communes, hésitant entre la révolte collective et l'individualisme consumériste. Groulx opte encore résolument pour une forme expérimentale et hétérogène associant couleur avec noir et blanc, voix off et intertitres, commentaires chantés, comédiens amateurs, références abondantes au cinéma antérieur. Le chapitre onze décrit Entre tu et vous (1969) qui poursuit de façon aussi radicale et pessimiste la réflexion sur la communication personnelle difficile dans une société dominée par les médias et la publicité. Un cinéaste quitte la ville avec sa compagne pour échapper à cet encerclement, mais il finit par vouloir incarcérer son amie. L'histoire est racontée de façon aussi hétéroclite et inattendue que dans le film précédent, entrecoupée de plans documentaires et de collages provocateurs. Le chapitre douze nous amène au film le plus explicitement engagé de Groulx, réalisé avec le politologue anarchiste Jean-Marc Piotte: 24 heures ou plus. Réalisé en 1972, le film ne sera diffusé qu'en 1976, les patrons de l'ONF en jugeant le contenu trop radical. Appelant à la grève générale et à la révolution, le film constitue un réquisitoire anticapitaliste reposant sur un montage et un commentaire d'exemples de répression. Groulx et Piotte y apparaissent dans des encadrés, lisant un commentaire marxiste sur les faits montrés de diverses façons: manchettes de journaux, films d'archives, entrevues avec diverses personnes, photo du syndicaliste Michel Chartrand qu'on entend dénoncer la situation de la classe ouvrière. Tombé en disgrâce, Groulx quitte alors l'ONF et doit travailler en usine pour survivre.

Jean-Claude Labrecque vient en aide à son ami Groulx en lui proposant la réalisation d'un autre film de commande pour l'Office du film du Québec. Place de l'équation (1973) déborde bien sûr la commande et constitue une étude très critique du système scolaire québécois, opposant une pédagogie participative aux vieilles traditions autoritaires. Le ton est moins radical que dans ses œuvres personnelles antérieures, mais le style est toujours reconnaissable. Le chapitre treize aborde un autre film politique fait au Mexique en collaboration avec une coopérative de paysans: Première question sur le bonheur (1977). Paul Beaucage n’a vraiment pas aimé ce film dont il 
trouve le didactisme primaire et le parti pris maladroitement défendu. Il est beaucoup plus enthousiaste pour le film suivant, qui fut le dernier de Groulx, dont la carrière s'est arrêtée en 1980 après un grave accident de voiture. $A u$ pays de Zom (1982) est une sorte de fable chantée sur les vertus et les vices d'un industriel égocentrique interprété par le chanteur d'opéra Joseph Rouleau. Divisé en neuf tableaux, le film est le plus brechtien de l'œuvre de Groulx, affichant fortement la distanciation et la critique acerbe du capitalisme. La conclusion du livre, plutôt brève, vante l'œuvre de Groulx comme pôle opposé du cinéma québécois devenu trop commercial.

Le livre de Beaucage constitue surtout une approche politique du travail de Groulx. Sur ce plan il explique assez bien les objectifs du cinéaste et les met en relief au travers des films, montrant l'évolution de Groulx d'une critique pessimiste de la société capitaliste vers un engagement révolutionnaire assumé, et la constance de son esthétique résolument éclectique qui cherche à déconstruire le média autant que le discours. C'est sur le plan théorique que le livre paraît plutôt faible. L'approche est surtout celle de Brecht et est certainement pertinente étant donné l'intérêt que lui portait Groulx, mais Beaucage ne nous dit presque rien sur Brecht hormis quelques citations qui sont à peine liées au propos du livre et à l'analyse des films. La réflexion du dramaturge et théoricien allemand n'est par ailleurs ni contextualisée ni mise en perspective, malgré les nombreuses études qui lui ont été consacrées. La bibliographie du livre est d'ailleurs rachitique, ne citant que des ouvrages généraux et anciens où ne figurent même pas les nombreuses études contemporaines sur le cinéma québécois. Pour introduire des concepts théoriques comme "expressionnisme» ou "documentaire» (p. 48 et p. 251), l'auteur renvoie aux deux lignes sur la question du Petit Robert. La plupart des citations du livre sont aussi impertinentes que superficielles. J'ai cité plus haut celle sur Bouguereau mais il y en bien d'autres aussi suaves, par exemple Victor Hugo: "la forme est du contenu qui remonte à la surface» (p. 114). Le livre est presque muet sur les détails biographiques et la diffusion de l'œuvre de Groulx: trois films lui ont été consacrés, qui ne sont mentionnés qu'en liste de fin de livre. L'auteur ne les commente pas, pas plus qu'il ne cite, commente ou discute les ouvrages généraux qui parlent de Groulx (Lever, Marshall, MacKenzie, Marsolais, etc.), pas plus qu'il n'a pensé à interroger les gens qui l'ont connu, ou fouiller les fonds d'archives qui auraient enrichi le livre.

On a l'impression que l'auteur a voulu faire un livre de vulgarisation pour expliquer et faire connaître l'œuvre singulière et magnifique de Groulx, et que cet objectif justifiait de négliger une exposition plus 
approfondie des prémisses de l'étude et de l'œuvre. Le lecteur appréciera peut-être la description politique, mais sera déçu par une approche théorique superficielle, et une recherche paresseuse.

Germain Lacasse

Université de Montréal 